\title{
Analysis of the interactions between close HVDC links inserted in an AC grid
}

\author{
Iulian Munteanu \\ École Centrale Nantes - IRCCYN \\ Nantes, France \\ Email: iulian.munteanu@ec-nantes.fr
}

\author{
Bogdan Marinescu \\ École Centrale Nantes - IRCCYN \\ Nantes, France \\ Email: bogdan.marinescu@ec-nantes.fr
}

\author{
Florent Xavier \\ Réseau de Transport d'Électricité \\ Versailles, France \\ Email: florent.xavier@ rte-france.com
}

\begin{abstract}
This paper is focused on interaction between two closed-connected high-voltage DC (HVDC) lines. This interaction is studied by employing electromagnetic transients (EMT)-based nonlinear modeling in MATLAB ${ }^{\circledR} /$ Simulink ${ }^{\circledR}$ software environment. In order to describe the mechanism behind the HVDCs interaction, both nonlinear time-domain simulations and modal analysis of the coupled HVDC links, using linearized version of the system, have been performed. System stability has been assessed and the path of interactions has been identified by computing the participations of various states in the oscillatory modes.
\end{abstract}

\section{INTRODUCTION}

Being flexible electrical power transmission structures able to convey large amount of power, high-voltage DC (HVDC) links may be used to connect two areas of the AC power grid to reinforce transmission capacity [1]. As two lines may be connected in areas having weak $\mathrm{AC}$ grid, their operation are likely to interfere due to geographic proximity, with potentially negative effects on their stability.

Parallel VSCs operation in AC grid context is studied in [2], and overall system stability is assessed by employing input-admittance modeling the converters. In [3] control solutions are proposed to diminish interaction between two close HVDCs using simplified phasor models. The present work develops the analysis of these interactions by using electromagnetic transients (EMT)-based modeling which captures the high-frequency VSC average behavior.

The approached system structure consists of two two-level HVDCs interconnected by means of a common AC power line, further denoted as ConAC - see Fig. 1. This is derived from the benchmark in [4], all AC lines and generators being simplified to impedances and infinite nodes. The simplification allows to better identify dynamic properties belonging exclusively to HVDCs coupling. Also, system behavior is assessed by means of modal analysis for continuous-time linear systems - [5], which offers a tool of determining the degree of participation of system states in a certain (oscillatory) mode. Originally used for stability issues in classical power system analysis, this approach may be applied to other types of complex systems, in which decoupling methods cannot be employed. A similar approach using multimodular converter (MMC)-based HVDC has been briefly presented in [6]. The present work extends system evaluation by thoroughly assessing states influence in shaping HVDC's interaction for various operating scenarios.
The paper is organized as follows. Section II goes on with HVDC line modeling aspects, while Section III presents nonlinear time-domain simulation and modal analysis results. Section IV concludes the paper.

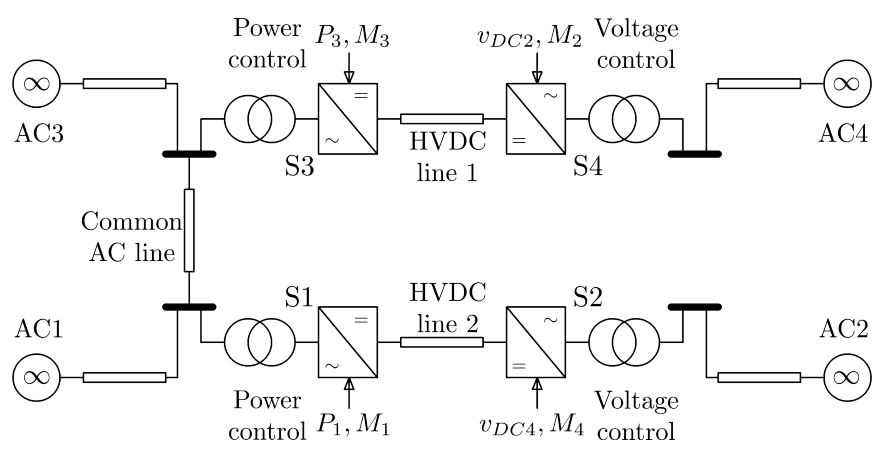

Fig. 1. Interconnection of two HVDC lines by means of an AC line.

\section{MODELING APPROACH}

\section{A. HVDC structure and assumptions}

VSC-HVDC transmission power structure (Fig. 2) corresponds to a monopolar structure with metallic return. It has two conversion stations that employ bidirectional 3-phased AC-DC power converters, interlinked by means of DC cables and connected to $\mathrm{AC}$ grids trough step-up transformers. VSC switched operation requires filtering elements on each station: capacitors on DC side and line reactors on AC side.

The following assumptions are considered. AC-DC converters are lossless and are switching instantaneously at a sufficiently high frequency; so the low-pass filter (habitually inserted between line reactor and transformer) is neglected. DC filter is reduced to capacitors, AC transformer has negligible magnetizing impedance and magnetic saturation phenomena are not considered. AC environment is modeled by AC infinite buses and variable series reactances; it is symmetrical and balanced. The common AC line is modeled as an RL circuit element. VSCs of HVDC link are operated in pulse-width modulation (PWM) in order to interchange averaged sinusoidal variables with AC grid. As this work is focused on the HVDCs low-frequency behavior, VSCs are modeled here by their averaged model, obtained by replacing converter switching functions with their sliding averages [7]. 


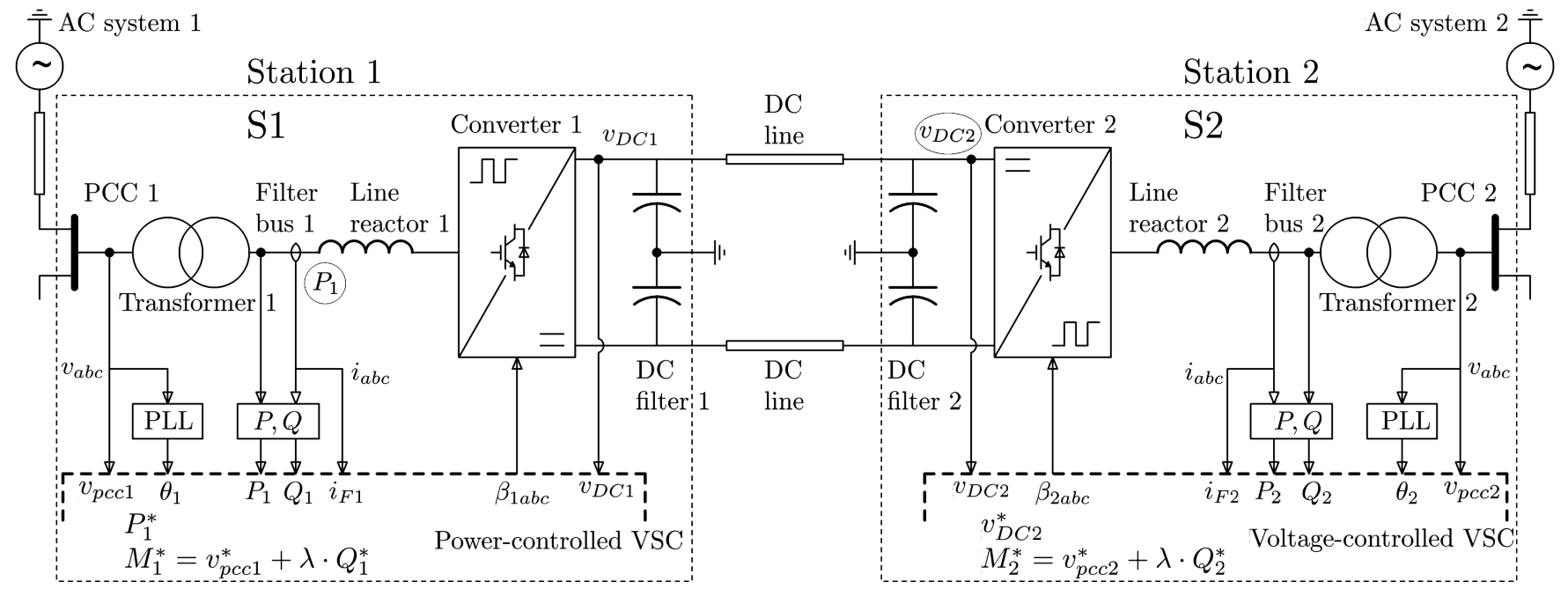

Fig. 2. HVDC transmission line overall control.

\section{B. $d q$ frame modeling}

The power subsystem (plant) model results from describing the electrical interactions between VSC and infinite node through passive circuit elements. First, one obtains an equivalent circuit by pushing the transformer primary inductance into the secondary (see Fig. 2 and transformer modeling in [7]); the whole AC system (including infinite nodes) becomes rated at VSC voltage value. Model is then described in rotating $d q$ frame linked to the infinite node; $d$ channel conveys active power and $q$ channel reactive power.

The plant model in this $d q$ frame is given by (1) - see [8],[9], where $i_{r d}$ and $i_{r q}$ are equivalent AC inductor current components, $i_{l d}$ and $i_{l q}$ are AC line components, $v_{r d}$ and $v_{r q}$ are the point of common coupling (PCC) voltage components, $E$ is the infinite node voltage amplitude, $\omega$ is AC system frequency and $v_{D C}$ is DC line voltage at the concerned VSC (capacitor filter voltage). $L_{r}$ and $r_{r}$ are reactor inductance and resistance, respectively, $L_{l}$ and $r_{l}$ are AC line inductance and resistance, respectively and $C$ is value of DC-side capacitors. $\beta_{r d}$ and $\beta_{r q}$ are plant control inputs. Voltage $v_{r d}$ is obtained by balancing currents $i_{r d}$ and $i_{l d}$ on a small resistive charge, $R_{D}: v_{r d}=\left(i_{l d}-i_{r d}\right) \cdot R_{D}$.

The same remark applies for channel $q$.

$$
\left\{\begin{aligned}
L_{r} \cdot \dot{i_{r d}} & =\omega L_{r} \cdot i_{r q}-0.5 v_{D C} \cdot \beta_{r d}+v_{r d}-r_{r} \cdot i_{r d} \\
L_{r} \cdot \dot{i_{r q}} & =-\omega L_{r} \cdot i_{r d}-0.5 v_{D C} \cdot \beta_{r q}+v_{r q}-r_{r} \cdot i_{r q} \\
L_{l} \cdot \dot{i_{l d}} & =\omega L_{l} \cdot i_{l q}-v_{d}+E-r_{l} \cdot i_{l d} \\
L_{l} \cdot \dot{i_{l q}} & =-\omega L_{l} \cdot i_{l d}-v_{q}-r_{l} \cdot i_{r q} \\
C \cdot v_{\dot{D C}} & =1.5\left(i_{r d} \cdot \beta_{r d}+i_{r q} \cdot \beta_{r q}\right)-i_{d c} \\
L_{d c} \cdot \dot{i_{d c}} & =v_{D C}-v_{D C r}-r_{d c} \cdot i_{d c}
\end{aligned}\right.
$$

Classical VSC control in the $d q$ frame linked to the PCC by means of a phase locked loop (PLL) [9], is used here, in which proportional-integral-law-based inner current control loops are cross-decoupled [8], [9]. On $d$ channel, either active power at PCC or DC-link voltage are controlled within an outer loop. On $q$ channel a mix between the PCC voltage and reactive power, $Q$ is controlled in a second outer loop - see [10]. This is here represented by variable $M=v_{P C C}+\lambda \cdot Q, \quad \lambda$ being the reactive power coefficient $(\mathrm{kV} / \mathrm{kVar})$.

$$
\left\{\begin{array}{l}
\beta_{d}=-K_{p c}\left(1+\frac{1}{T_{i c} s}\right)\left(\frac{i_{d}^{*}}{T_{i c} s+1}-i_{d}\right)+\frac{2 \omega L_{r}}{v_{D C}} \cdot i_{q} \\
\beta_{q}=-K_{p c}\left(1+\frac{1}{T_{i c} s}\right)\left(\frac{i_{q}^{*}}{T_{i c} s+1}-i_{q}\right)-\frac{2 \omega L_{r}}{v_{D C}} \cdot i_{d} \\
i_{d}^{*}=\frac{1}{T_{i P} s} \cdot\left(P^{*}-P\right) ; i_{q}^{*}=\frac{1}{T_{i M} s} \cdot\left(M^{*}-M\right) \\
P=1.5\left(v_{d} \cdot i_{d}+v_{q} \cdot i_{q}\right) ; Q=1.5\left(v_{d} \cdot i_{q}-v_{q} \cdot i_{d}\right)
\end{array}\right.
$$

The overall control law expressed in $d q$ frame linked to PCC is given by (2) - see [6], [8] - where $i_{d}$ and $i_{q}$ are measured reactor current components, $v_{d}$ and $v_{q}$ are voltages measured at PCC, $P$ and $Q$ are PCC active and reactive power components and $s$ is the Laplace operator. Variables $\left\langle\cdot *^{*}\right\rangle$ denote the associated setpoints. $K_{p c}$ and $T_{i c}$ are the proportional gain and integrator constant of PI current controllers, $T_{i P}$ is the active power controller integrator constant and $T_{i M}$ is the reactive power controller integrator constant. The third equation of (2) is valid only for the power-controlled station/VSC. Stations with (DC) voltage control employ (3) instead.

$$
i_{d}^{*}=K_{p v}\left(1+\frac{1}{T_{i v} s}\right) \cdot\left(v_{D C}^{*}-v_{D C}\right)
$$

In order to render variables from the control and power structures compatible, one must make a rotation transform from the PCC-linked frame to the infinite-node-linked frame. This is done by using the Kron's transform [11], which uses the phase shift between the two frames, denoted by $\delta$ : 


$$
\left\{\begin{aligned}
i_{d} & =i_{r d} \cdot \cos \delta-i_{r q} \cdot \sin \delta \\
i_{q} & =i_{r d} \cdot \sin \delta+i_{r q} \cdot \cos \delta \\
\beta_{r d} & =\beta_{d} \cdot \cos (-\delta)-\beta_{q} \cdot \sin (-\delta) \\
\beta_{r q} & =\beta_{d} \cdot \sin (-\delta)+\beta_{q} \cdot \cos (-\delta)
\end{aligned}\right.
$$

PCC phase angle is extracted from its voltages by using a 3-phase PLL. PLL angle serves for all computations in the station control system, but its value is delayed with respect to the PCC angle. The overall dynamic behavior of the controlled VSC, is thus impacted - see [12]. In this work, a new PLL structure has been used - see Fig. 3, which has exactly the same dynamics as $1^{\text {th }}$ order classical PLL. It outputs not only PCC voltage components $v_{d}$ and $v_{q}$, but also the phase shift $\delta$. PLL dynamics intervene twice: in $i_{d}$ and $i_{q}$ measurement and in $\beta_{r d}$ and $\beta_{r q}$ actuation by means of angle $\delta$.

Further, stations S1 and S3 (see Fig. 1) are modeled by using (1) and (2), i.e., they are power-controlled, and stations S2 and S4 use (1) and (2), where the third equation is replaced by (3), i.e., they are voltage-controlled. ConAC line is modeled as a RL line, by using the equations similar to the two first equations from (1).

The control system of a single HVDC line is tuned to obtain pertinent performances for $i_{d}$ and $i_{q}$ : damping at around 0.65 - 0.85 and time constant $5 \mathrm{~ms}$ for the inner loops, for typical scenarios and operating points. Time constant for outer loops is around $17 \mathrm{~ms}$ for $v_{D C}$ control loop and $20-30 \mathrm{~ms}$ for active power $P$ and variable $M$. All these outer loops have variable damping depending on grid equivalent short-circuit ratio (SCR) and on the operating point.

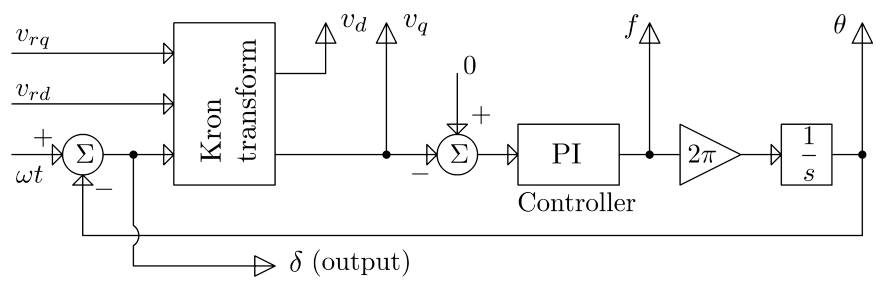

Fig. 3. PLL structure using Kron's transform as phase detector; steady-state value of $\delta$ nullifies voltage $v_{q}$.

\section{System linearization and modal analysis}

All system states (integrators) are labeled with suitable keywords (e.g., "int PI id2" means the state associated with the $i_{d 2}$ current controller integrator). Linearization is done by using the Linear analysis tool available in MATLAB ${ }^{\circledR} /$ Simulink ${ }^{\circledR}$ software around a suitable steady-state operating point. In all results in this section the input is chosen as $M_{1}^{*}$ (the setpoint) and the output as $M_{1}$, although any of variables $P_{k}$ and $M_{k}$ with $k=1 . .4$, may be set as output.

Modal analysis for continuous-time linear time-invariant system starts with the state matrix, denoted by $A$, previously obtained by linearization. Then, normalized right eigenvectors $v_{i}$ and left eigenvectors $w_{i}$, which verify that

$$
\left\{\begin{array}{c}
A \cdot v_{i}=v_{i} \cdot \lambda_{i} \\
w_{i} \cdot A=\lambda_{i} \cdot w_{i},
\end{array}\right.
$$

with $w_{i} \cdot v_{j}=0$ for $i \neq j$ and $w_{i} \cdot v_{j}=1$ for $i=j-$ see [5] are determined, where $\lambda_{i}$ are the state matrix $A$ eigenvalues.

The participation factor of variable $x_{j}$ in mode $i$ is computed as the product between elements $j$ of vectors $w_{i}$ and $v_{i}$ - see [5] for quick reference :

$$
p_{j i}=\left(w_{i}\right)_{j} \cdot\left(v_{i}\right)_{j} \cdot
$$

One determines participation factors for all state variables and for all system modes, resulting values form the so-called participation factor matrix (square matrix of system dimension) [5]. Absolute values of participation factors are further used, in order to compare variables that intervene in a certain mode. The modes of interest are chosen by using damping criterion i.e., the ones having the damping under a certain threshold, in order to emphasize system oscillations/couplings.

\section{ANALYSIS RESULTS}

\section{A. Nonlinear time-domain simulations}

In all subsequent scenarios, only parameters/setpoints of stations S1 and S3 will change; stations S2 and S4 setpoints are $v_{D C 2}=v_{D C 4}=640 \mathrm{kV}$ and $M_{2}=M_{4}=332 \mathrm{kV}$. Their associated SCR is always constant, at $\approx 5$. Also, the values considered for ConAC line length correspond to the initiallystated "close" HVDC lines context.

The first scenario consists in injecting active power values $P_{1}=-500 \mathrm{MW}$ and $P_{3}=-250 \mathrm{MW}$ and maintaining $M_{1}=$ $M_{3}=332 \mathrm{kV}$ (i.e., at their rated values), for various ConAC line lengths - see Figs. 4 a) and b). Both S1 and S3 have the same $\mathrm{SCR}=5$ value. A large step of $M_{1}$ setpoint $(\approx 500 \mathrm{MVar}$ of reactive power), has been imposed.

Fig. 4 a) presents $M_{1}$ evolutions (local control loop voltage tracks setpoint variation), and Fig. 4 b) $M_{3}$ evolutions corresponding control loop rejects the perturbation. System's dynamic behavior has two main components: a damped behavior whose frequency becomes smaller as ConAC line length decreases, and a second undamped behavior, whose frequency is around $20 \mathrm{~Hz}$ and which is the target of the subsequent modal analysis.

In Fig. 4 c) a detail of $M_{1}$ (black) and $M_{3}$ (red) evolutions at a $M_{1}$ setpoint step (ConAC is of $100 \mathrm{~km}$ ) is shown. The same transient dynamics can be noted on both outputs - oscillations have the same frequency, the same damping and the same phase, suggesting that the two input-output transfer channels are coupled, as their oscillations are due to the same mode.

Fig. 4 d) shows $M_{3}$ evolution at a step in $M_{1}$ setpoint, within a different scenario, with various SCR values at both stations S1 and S3 and constant ConAC line length at $150 \mathrm{~km}$. $\mathrm{S} 1$ and S3 setpoints are the same as in the previous scenario. The system is less damped as SCRs values at both S1 and S3 are smaller (the grid is weaker). If one continues to reduce SCR values, the system becomes unstable. 

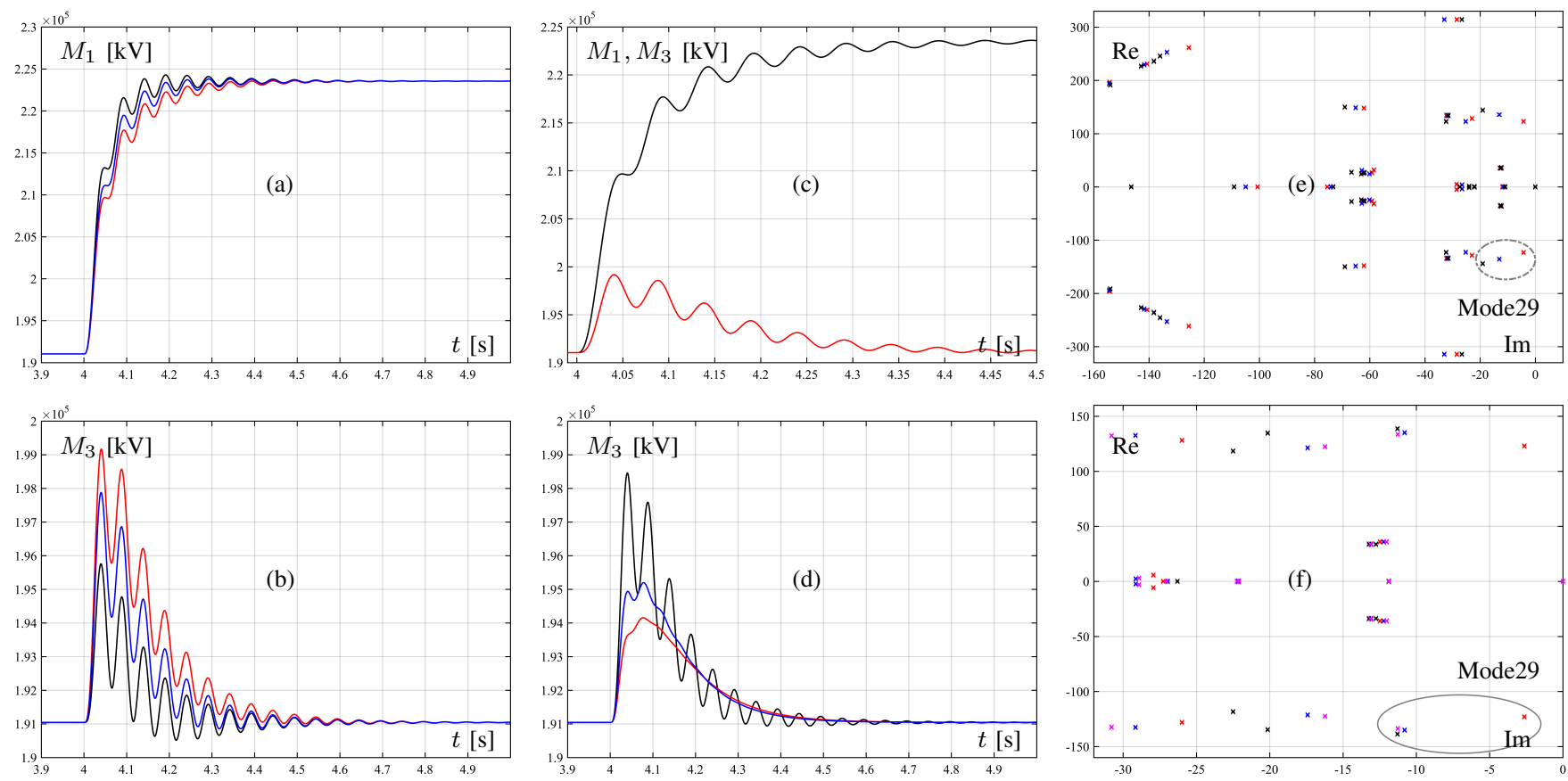

Fig. 4. a) and b): system response at $M_{1}$ stepoint step variation, for various ConAC line lengths black $-500 \mathrm{~km}$, blue $-200 \mathrm{~km}$, red $-100 \mathrm{~km}$, (a) $M_{1}$ evolution, b) $M_{3}$ evolution); c): detail of $M_{1}$ (black) and $M_{3}$ (red) evolutions at a $M_{1}$ setpoint step (ConAC is of $100 \mathrm{~km}$ ); d): $M_{3}$ evolution at $M_{1}$ stepoint step variation, for various SCR values (black -7.5 , blue -5 , red -3.5 ); e): system pole-zero maps for various SCR values: black -5 , blue -4 , red -3 ; f): system pole-zero maps for various $P_{1}$ and $P_{3}$ setpoints; red: $P_{1}=-500 \mathrm{MW}, P_{3}=-600 \mathrm{MW}$, blue: $P_{1}=-500 \mathrm{MW}, P_{3}=+600 \mathrm{MW}$, magenta: $P_{1}=+500 \mathrm{MW}, P_{3}=-600 \mathrm{MW}$, black $P_{1}=+500 \mathrm{MW}, P_{3}=+600 \mathrm{MW}$.

TABLE I

Mode 29 PARAMETERS VARIATIONS: LEFT SIDE - WITH SCR VALUES, RIGHT SIDE - WITH $P_{1}$ AND $P_{3}$ VALUES

\begin{tabular}{c|cc||c|cc} 
SCR & Freq. $[\mathrm{Hz}]$ & Damp. & $P_{1}, P_{3}[\mathrm{MW}]$ & Freq. [Hz] & Damp. \\
\hline 3 & 19.6 & 0.034 & $-500,-600$ & 19.57 & 0.021 \\
4 & 21.6 & 0.096 & $-500,+600$ & 21.5 & 0.08 \\
5 & 22.94 & 0.131 & $+500,-600$ & 21.27 & 0.084 \\
- & - & - & $+500,+600$ & 22.08 & 0.081 \\
\hline
\end{tabular}

\section{B. Modal analysis}

For each scenario the nonlinear system is linearized around the associated steady-state operating point, by using MATLAB ${ }^{\circledR} /$ Simulink ${ }^{\circledR}$; thus, a 62-state linear model is obtained. Modal analysis algorithm - based upon equations (5) and (6) - is applied and the previously-observed oscillatory mode (see Fig. 4) is identified. It will be further referred to as Mode 29.

The first scenario involves modal analysis for three different SCR values at stations S1 and S3. Steady-state active power values are $P_{1}=-500 \mathrm{MW}$ and $P_{3}=-250 \mathrm{MW}, M$ variables $M_{1}=M_{3}=332 \mathrm{kV}$ and ConAC line length is $150 \mathrm{~km}$.

Table I (left side) shows Mode 29 main parameters for the three SCR values. Frequency has not an important variation, but the damping is severely decreasing with SCR, thus affecting the system stability. Fig. 4 e), which contains the system pole's evolution as SCR decreases (only low-frequency complex-conjugated poles have been plotted), shows the same
TABLE II

STATES INFLUENCES IN Mode 29 FOR TWO VALUES OF SCR.

\begin{tabular}{cc|cc} 
SCR $\rightarrow$ & 4 & SCR $\rightarrow$ & 3 \\
\hline State name & Participation & State name & Participation \\
\hline int PI iq1 & 0.09 & int PLL1 & 0.15 \\
int PLL1 & 0.09 & int PI iq1 & 0.14 \\
int PI iq3 & 0.09 & int PLL3 & 0.13 \\
int PLL3 & 0.08 & int PI iq3 & 0.13 \\
int PI id1 & 0.06 & int PI id3 & 0.09 \\
Reactor 1/int2 & 0.07 & int PI id1 & 0.1 \\
Reactor 3/int2 & 0.05 & Reactor 1/int2 & 0.09 \\
int PI id4 & 0.05 & Reactor 3/int2 & 0.07 \\
int PLL4 & 0.05 & int PI PLL1 & 0.06 \\
int PI iq4 & 0.05 & AC line 1/int2 & 0.05 \\
\hline
\end{tabular}

trend. Table II contains ranking of most participative system states in Mode 29 for two values of SCR. Variables belonging to $\mathrm{S} 1$ - from the HVDC link 1 - and S3 - from the HVDC link 2 - have the most important contribution to the concerned mode. This proves that the two HVDCs are interacting.

The second scenario concerns modal analysis for various active power values (positive/power draw or negative/power injection) required for stations $\mathrm{S} 1$ and $\mathrm{S} 3$. The set-up presumes a ConAC line of $150 \mathrm{~km}$, both $\mathrm{S} 1$ and S3 maintain variables $M_{1}$ and $M_{3}$ at their rated values (i.e., $332 \mathrm{kV}$ ); SCR is the same for $\mathrm{S} 1$ and $\mathrm{S} 2$, i.e., 3.5. Stations $\mathrm{S} 2$ and $\mathrm{S} 4$ follow operation of stations $\mathrm{S} 1$ and $\mathrm{S} 3$ respectively, by regulating DC voltage at the rated value $(640 \mathrm{kV})$.

Table I (right side) shows Mode 29 parameters variation 
TABLE III

States With LaRgest Participation IN Mode 29 For VARIOUS $P_{1}$ AND $P_{3}$ VAlues.

\begin{tabular}{rl|rl|rl|rc}
\hline$P_{1}=-500, P_{3}=-600$ & {$[\mathrm{MW}]$} & $P_{1}=+500, P_{3}=+600$ & {$[\mathrm{MW}]$} & $P_{1}=+500, P_{3}=-600$ & {$[\mathrm{MW}]$} & $P_{1}=-500, P_{3}=+600$ & {$[\mathrm{MW}]$} \\
\hline State name & Part. & State name & Part. & State name & Part. & State name & Part. \\
\hline int PLL1 & 0.13 & int PI iq4 & 0.07 & int PI id2 & 0.17 & int PI id4 & 0.14 \\
int PLL3 & 0.13 & int PI id4 & 0.07 & int PLL2 & 0.15 & int PLL4 & 0.12 \\
int PI iq3 & 0.13 & int PLL4 & 0.07 & int PI iq2 & 0.14 & int PI iq4 & 0.12 \\
int PI iq1 & 0.12 & int PI id2 & 0.06 & int PLL3 & 0.1 & Reactor 4/int 2 & 0.09 \\
int PI id3 & 0.1 & int PLL2 & 0.06 & int PI iq3 & 0.1 & int PLL1 & 0.07 \\
int PI id1 & 0.09 & int PI iq2 & 0.05 & Reactor 2/int 2 & 0.1 & int PI iq1 & 0.07 \\
Reactor 3/int 2 & 0.08 & Reactor 4/int 2 & 0.05 & Reactor 2/int 1 & 0.09 & Reactor 4/int1 & 0.07 \\
Reactor 1/int 2 & 0.08 & Reactor 2/int 2 & 0.04 & AC line 2/int1 & 0.07 & Prefilter id4 & 0.06 \\
AC line 3 /int 2 & 0.05 & int PLL1 & 0.04 & Prefilter id2 & 0.07 & AC line 4 /int1 & 0.06 \\
int PI PLL3 & 0.05 & int PI iq1 & 0.04 & Reactor 3/int2 & 0.07 & Reactor 1/int 2 & 0.05 \\
\hline
\end{tabular}

with $P_{1}$ and $P_{3}$ values. Damping of Mode 29 is lowest when both $\mathrm{S} 1$ and $\mathrm{S} 3$ inject power in $\mathrm{AC}$ grid. This is also visible on Fig. $4 \mathrm{f}$ ), showing variation of pole associated with Mode 29 with respect to power levels.

Ranking of first ten states participating in Mode 29 are shown in Table III. The inner loop (current) controllers states, including PLLs (either the PLL integrator or its PI controller), have the most important participation in this mode. Note also, that these states belong to stations that inject active power. For example, ranking in column 3 of Table III, corresponds to case where S1 draws power and S3 injects power from/in their AC grids. Therefore, S2 injects power and S4 draws power in/from their AC grids in order to maintain DC voltage at the prescribed value. States belonging to stations S2 and S3 are the most participative. This remark holds for all cases (see Table III). Also, notice that the coupling mode is systematically better damped in scenarios where powercontrolled stations (S1 and S3) draw power - see the right side of Table I.

Note that in all cases the states belonging to the ConAC line do not significantly intervene in Mode 29. Also, states belonging to VSC reactors, AC lines and low-level loop prefilters - see equations (2) - may also be significantly involved in the participation mix.

\section{CONCLUSION}

This paper has dealt with analysis of two close-connected HVDC lines by using high-fidelity models in $d q$ frame.

A potentially inconvenient interaction, due to HVDCs coupling, has been highlighted by time-domain simulation. Also, modal analysis in various scenarios has revealed that low-level controllers states and PLL states significantly participate in the coupling oscillatory mode. The damping of coupling mode may be under $5 \%$ if both AC-linked stations inject active power into the grid. This damping becomes smaller as AC grid SCRs decrease.

Further work will focus to study interaction between HVDC links operating into richer AC environment which includes synchronous generators. Also, based upon this analysis, corrective control actions will be proposed.

\section{APPENDIX. SYSTEM PARAMETERS}

- AC system: frequency $50 \mathrm{~Hz}$, rated RMS voltage $400 \mathrm{kV}$,

- AC line: resistance $0.03 \Omega / \mathrm{km}$, inductance $0.79 \mathrm{mH} / \mathrm{km}$,

- Transformer: secondary RMS voltage $230 \mathrm{kV}$, ratio $1.74, R_{p r}=$ $0.4 \Omega, L_{p r}=38 \mathrm{mH}, R_{\text {sec }}=0.132 \Omega, L_{\text {sec }}=12.6 \mathrm{mH}$,

- Line reactor: impedance $25.3 \mathrm{mH}$, resistance $0.08 \Omega$,

- DC line: voltage $640 \mathrm{kV}$, capacitor $220 \mu \mathrm{F}$, resistance 13.9 $\mathrm{m} \Omega / \mathrm{km}$, impedance $0.159 \mathrm{mH} / \mathrm{km}$, length $200 \mathrm{~km}$,

- VSCs: active power $1000 \mathrm{MW}$, reactive power $Q \in$ $\{-500 ;+400\}$ MVAr, maximum active current \pm 4 kA, maximum reactive current $\pm 1.6 \mathrm{kA}$,

- Control parameters: $\lambda=13 \mathrm{e}-6 \mathrm{~V} / \mathrm{VAr}, T_{i c}=6.6 \mathrm{~ms}, K_{p c}=0.6 \mathrm{e}-$ $4 \mathrm{~A}^{-1}, K_{t c}=5 \cdot K_{t p}=500, K_{p v}=0.034 \mathrm{~A} / \mathrm{V}, T_{i v}=25$ $\mathrm{ms}, T_{i m}=0.2845 \mathrm{~s}, T_{i p}=7.04 \mathrm{~ms}$.

\section{REFERENCES}

[1] N. Flourentzou, V. G. Agelidis, and G. D. Demetriades, "VSC-Based HVDC Power Transmission Systems: An Overview," IEEE Trans Power Electron vol. 24, no. 3, pp. 592-602, 2009.

[2] A. Bayo-Salas, J. Beerten, J. Rimez, and D. Van Hertem, "Impedancebased stability assessment of parallel VSC HVDC grid connections," in the $11^{\text {th }}$ IET International Conference on AC and DC Power Transmission, 10-12 February, Birmingham, UK, pp. 1-9, 2015.

[3] L. Arioua and B. Marinescu, "Multivariable control with grid objectives of an HVDC link embedded in a large-scale AC grid," Electrical Power and Energy Systems, vol. 72, 99-108, 2015.

[4] S. Henry, O. Despouys, R. Adapa and al., "Influence of Embedded HVDC Transmission on System Security and AC Network Performance," Cigre JWG C4/B4/C1.604, 2013.

[5] G. Rogers, "Power system oscillations," Springer Science+Business Media, New York, USA, 2000

[6] Saad HA, "Modeling and simulation of a VSC-MMC HVDC link" (in French: Modélisation et simulation d'une liaison HVDC de type VSCMMC), PhD. Thesis, University of Montréal, Canada, 2015.

[7] R. W. Erikson and D. Maksimović "Fundamentals of power electronics, 2nd edn.," Kluwer, New York, USA, 2001.

[8] W. Wang, A. Beddard, M. Barnes, and O. Marjanovic, "Analysis of Active Power Control for VSC-HVDC," IEEE Trans. on Power Delivery, vol. 29, no. 4, pp:1978-1988, 2014.

[9] S. Bacha, I. Munteanu, A.I. Bratcu, "Power Electronic Converters Modeling and Control," Springer, London, UK, 2014.

[10] RTE, France "Voltage regulation and constructive capacities in reactive power of generation installations," Reference technical documentation, 2016. http://clients.rte-france.com/lang/fr/visiteurs/mediatheque_ client/offre.jsp

[11] K.R. Padiyar "Analysis of subsynchronous resonance in power systems," Springer Science+Business Media, New York, USA, 1999.

[12] B. Wen, D. Boroyevich, P. Mattavelli, Z. Shen, and R. Burgos "Influence of Phase-Locked Loop on Input Admittance of Three-Phase VoltageSource Converters," in the $23^{\text {th }}$ Applied Power Electronics Conference and Exposition (APEC),17-21 March, Long Beach, CA, USA, pp. 897904, 2013. 